\title{
Une première histoire des réiugiés juifs de Belgique (1945-1948)
}

\begin{abstract}
Dans son ouvrage, La filière des ombres, Jacques DÉOM' s'intéresse à un épisode singulier de l'immédiat aprèsGuerre, à savoir l'acheminement vers la Palestine, alors placée sous le mandat de la Grande-Bretagne, de Juifs en provenance des camps allemands tran-

L'auteur : Pr. Dr Geoffrey GRANDJEAN est Chargé de cours à la Faculté de Droit, de Science politique et de Criminologie de l'Université de Liège.
\end{abstract}

sitant par la Belgique. La méconnaissance de cet épisode s'explique aisément selon l'auteur : " par rapport à la Seconde Guerre mondiale, l'immédiat après-Guerre est une période de transition, passée rapidement en revue ${ }^{2}$, notamment par les historiens. II s'agit en fait d'une immigration "illégale " dans la mesure où elle a été mise en place en violation des quotas imposés par la Grande-Bretagne. Ce mouvement d'immigration porte le nom d'Alyah Bet. Pour mener à bien son étude chronologique, l'auteur a eu l'occasion de consulter une série d'archives israéliennes, britanniques et belges. Au total, entre 1934 et 1948, plus de 106.000 Juifs ont été transférés «illégalement " par voie maritime (quatre-vingts bateaux) en Palestine ou à Chypre.

Dans son ouvrage, le philosophe s'intéresse plus précisément à trois bateaux et à leur voyage respectif vers la Palestine : le Tel Chay, le Hachayal haivri et le Theodor Herzl. Avant de revenir sur cette triple aventure, il convient de rappeler le contexte politique belge de l'époque : "dans l'immédiat après-Guerre, la Belgique enregistre, dans le chaos ambiant, un afflux de migrants d'Europe centrale et orientale. Pour des raisons essentiellement budgétaires, le gouvernement d'Achille VAN ACKER ${ }^{3}$ s'emploie d'emblée à limiter l'immigration. Seuls peuvent être admis à s'établir en Belgique ceux dont la présence

' Jacques DÉOM est licencié en philosophie et en philologie biblique de l'Université catholique de Louvain. Il est actuellement chercheur à la Fondation de la Mémoire contemporaine.

2 Entretien avec Jacques DÉOM, jeudi 28 mai 2015, Bruxelles, Fondation de la Mémoire contemporaine.

3 Achille VAN ACKER (1898-1975) est un homme politique belge, membre du parti socialiste. Député, il a été à plusieurs reprises Premier ministre (1944-1945, 1945-1946, 1946, 1954-1958) avant de prendre la présidence de la Chambre des Représentants. Il est considéré comme le père de la sécurité sociale. DUMONT GeorgesHenri, Chronologie de la Belgique. De 1830 à nos jours, Bruxelles, Le Cri, coll. « Histoire », 2003, p. 238. 
s'avère utile à la communauté "4. La Belgique a dès lors fermé les yeux sur le mouvement d'émigration vers la Palestine, quand bien même des craintes britanniques se font ressentir.

Le Tel Chay est le premier bateau à partir du port de La Ciotat (France), le 17 mars 1946. II a inauguré une série de quatorze départs qui se sont terminés en avril 1948.

Le Hachayal haivri est le deuxième bateau à partir du port d'Anvers (Belgique), le 14 juillet 1946. Le Hachayal haivri a effectué le deuxième trajet le plus long (après celui réalisé par un bateau parti de Suède) sur les quelques quatre-vingts convois d'immigrants clandestins.

Le Theodor Herzl est le troisième bateau à partir du port de Sète (France), le 2 avril 1947. Ce bateau a compté plus de 2.600 rescapés dont 1.500 d'entre eux environ ont transité par la Belgique. Ce voyage a fait l'objet d'une grande médiatisation. Deux journalistes sont ainsi montés à bord: Moshe PEARLMAN, britannique, et François-Jean ARMORIN, français, lequel a offert un récit de ce voyage ${ }^{5}$. Repéré par les Autorités britanniques, l'assaut du bateau fit deux morts et vingtquatre blessés. L'ensemble des passagers ont été expédiés sur l'île de Chypre.

Pour chaque voyage, l'auteur dresse une description détaillée permettant de mieux cerner ce périple peu connu de l'histoire mondiale. Et pour cause, l'auteur a disposé de rapports détaillés, heure par heure, des voyages de ces bateaux. Par contre, peu d'archives détaillent les étapes qui précèdent. "La tranche belge de l'Alyah Bet est mal documentée ${ }^{6}$, selon l'auteur et, d'une manière générale, «beaucoup de choses ont été improvisées à l'époque " ${ }^{7}$, rendant les traces écrites moins nombreuses. Cela donne parfois au livre un "caractère allusif " $^{8}$, comme le fait remarquer Jacques DÉOM, qui reconnaît également que l'ouvrage ne prétend pas à l'exhaustivité mais constitue davantage une évocation, compte tenu de l'état des informations mises à sa disposition et dont il a pu prendre connaissance.

Les récits de ces voyages sont entrecoupés d'une série d'esquisses, à la fois de la société belge et européenne mais également

${ }^{4}$ DÉOM Jacques, La filière des ombres. L'odyssée des réfugiés juifs Belgique - Palestine (1948-1948), Bruxelles, Fondation de la Mémoire contemporaine, 2015, p. 53.

${ }^{5}$ ARMORIN François-Jean, Des Juifs quittent l'Europe, Paris, Julliard, 1990 (réédition de 1948), 265 p.

${ }^{6}$ Entretien avec Jacques DÉOM, jeudi 28 mai 2015, Bruxelles, Fondation de la Mémoire contemporaine. 7 Ibid.

${ }^{8} \mathrm{lbid}$. 
du contexte international. On ne peut en effet comprendre ces mouvements migratoires sans élargir la focale de la recherche. On comprend ainsi premièrement mieux les raisons ayant poussé une série de Juifs, dans le chaos de l'immédiat après-guerre, se retrouvant sur le sol belge, animés "du désir de n'y faire qu'un séjour limité et de pouvoir gagner dans les plus brefs délais des cieux plus riants que ceux qui pèsent sur la vieille Europe $"$.

On cerne deuxièmement mieux toute la difficulté de franchir les frontières au lendemain de la Deuxième Guerre mondiale, notamment la frontière allemande. À cet égard, «le travail de la Brichah (littéralement : fuite, au sens d'exfiltration) n'est guère possible qu'en recourant à des voies plus ou moins légales, et immanquablement tortueuses $»^{10}$.

Troisièmement, le processus de migration a nécessité la mise en place de "centres d'habilitation " (hachsharot) qui sont des institutions communautaires de formation professionnelle, culturelle et linguistique des jeunes mises sur pied par le mouvement sioniste afin de les préparer à l'immigration en Palestine". Ces centres permettent à ces jeunes "de se retrouver comme juifs non honteux "12. Parmi ceux-ci, figure notamment l'École artisanale et agricole du Bahad, à Marquain, près de Tournai, qui favorise des conditions de vie normales pour les jeunes en leur assurant une éducation adéquate. Il y a également le hachshara de Kessel-Lo, dans la banlieue de Louvain dont l'encadrement est directement conçu dans la perspective de l'immigration en Palestine :

Inauguré en mai 1946, Kessel-Lo constitue un centre éducatif pour jeunes de 14 à 17 ans se destinant à l'immigration en Palestine et son style de vie correspond le plus fidèlement possible au régime en honneur dans les centres d'entraînement de Palestine pour l'âge correspondant : $1 / 2$ jour d'études, $1 / 2$ jour d'éducation professionnelle. Les garçons s'y préparent aux métiers des champs au moyen de travaux pratiques et de cours théoriques de chimie, physique, botanique, etc. L'initiation professionnelle des filles comprend des cours de couture et de coupe, des travaux de ravaudage et de tricot. Les matières juives incluent histoire du peuple juif et de la colonisation en Palestine, Bible. À quoi s'ajoutent les matières générales: histoire, géographie, anglais et des excursions $[\ldots]^{13}$.

- DÉom Jacques, La filière des ombres. L’odyssée des réfugiés juifs Belgique - Palestine (1948-1948), op. cit., p. 105.

10 Ibid., p. 113.

"Ibid., p. 121.

${ }^{12}$ Entretien avec Jacques DÉOM, jeudi 28 mai 2015, Bruxelles, Fondation de la Mémoire contemporaine.

${ }^{13}$ DÉOM Jacques, La filière des ombres. L'odyssée des réfugiés juifs Belgique - Palestine (1948-1948), op. cit., p. 133. 
Quatrièmement, on comprend mieux «l'aventure » qu'a constituée, notamment en termes administratifs, l'Alyah Bet. Jacques DÉOM nous livre ainsi une anecdote plusieurs fois rapportée par Fera PERELMAN ${ }^{14}$ concernant l'embarquement à bord du Hachayal haivri, qui témoigne de la dimension «illégale » de la migration:

\begin{abstract}
Les passeports des émigrants étaient collectifs et revêtus de visas de sortie belges, ainsi que de visas à destination du Panama. Le commissaire de la Sûreté et ses aides siégeaient à une table près du bateau et vérifiaient, un à un, les noms des gens qui montaient à bord». Fela PERLEMAN obtient de les rejoindre : "Tout allait bien : les gens répétaient à la perfection leurs noms et dates de naissance apprises par cœur la veille, jusqu'au moment où, à ma plus grande stupéfaction, quelqu'un se présente porteur d'un nom déjà employé par un autre. J'ai pu convaincre le commissaire que de telles 'coïncidences' étaient possibles... Chez nous, Juifs, il peut arriver que deux personnes aient le même nom, car quand un homme célèbre meurt, on donne son nom aux enfants qui naissent le jour de sa mort... Mais que lui dire quand se succèdent vingtcinq cas du même genre? Sur ma suggestion, pour ne pas retarder le départ du bateau, le commissaire consentit à laisser passer les cas 'en règle' et à ne retenir que les cas 'douteux'. Alors que, à l'exception de ces quelques malheureux consignés, tout ce monde se trouvait à bord, nous proposâmes de faire une pause autour d'une tasse de café. Entretemps, je fis signe à Chanania WEISSELBERG, qui attendait mes ordres, de faire monter en notre absence les 'consignés', si bien que lorsque nous revînmes, il n'y avait plus personne à terre. Plutôt que de faire redescendre tout ce monde aux fins de vérification, le commissaire, mis devant le fait accompli, autorisa le départ du bateau. Et nous laissâmes aux autorités du 'Panama' le soin de découvrir les 'resquilleurs'. Enquête faite, il s'avéra que l'on avait par erreur fait circuler dans deux camps la même liste de trente noms fictifs... " $^{15}$.
\end{abstract}

Malgré cette anecdote, l'auteur souligne que, en Belgique, les opérations relatives à l'Alyah Bet ont été réalisées " quasi légalement " $^{16}$, notamment par l'action de Fera PerELMAN. À cet égard, on peut notamment relever le rôle de l'association sans but lucratif Jewish Refugees Welfare Society (JRWS) - Fuvre de Protection des Réfugiés juifs (OPRI) - «instrument légal au service de la clandesti-

\footnotetext{
${ }^{14}$ Fera Perelman (1909-1991) est la cheville ouvrière de l'Alyah Bet en Belgique. Elle a notamment joué un rôle d'accueil des enfants juifs au lendemain de la Deuxième Guerre mondiale et a été à la base de la création des écoles maternelles Nos petits, en mai 1942. Ces écoles permettent de contrecarrer l'interdiction scolaire qui frappe les enfants juifs suite aux mesures allemandes. DÉom Jacques, La filière des ombres. L’odyssée des réfugiés juifs Belgique - Palestine (1948-1948), op. cit., pp. 39-49. ${ }^{15}$ Ibid., p. 154.

${ }^{16}$ Entretien avec Jacques DÉOM, jeudi 28 mai 2015, Bruxelles, Fondation de la Mémoire contemporaine.
} 
nité $1^{17}$ - constituée en 1947, qui plaide publiquement la cause des malheureux réfugiés juifs et dont l'objectif est clairement de mettre fin à leur détresse en mettant en place des moyens leur permettant de sétablir définitivement dans des pays qui souhaiteraient les accueillir.

Ces esquisses sont cinquièmement l'occasion de cerner des trajectoires individuelles comme celle de Maurice SzMIDT, né en Pologne en 1925, arrivé enfant en Belgique et parti en exode dans un petit village du Midi de la France. Victime d'une grande rafle dans la zone dite libre, le 28 août 1942, il est emmené à Drancy avant d'être déporté et de connaître plusieurs camps nazis : Johannsdorf, Kochanowitz, Borsigwerk, Blechhammer et, au terme d'une marche de la mort de 250 kilomètres, Gross-Rosen et Buchenwald. Libéré, il rentre en France où il retrouve sa famille à Lyon. Dans l'immédiat après-guerre, il rejoint un groupe sioniste afin d'immigrer en Palestine. Sa volonté se réalise grâce à l'alyah illégale en embarquant à bord du Hachayal haivri $^{18}$.

Cet ouvrage est par ailleurs l'occasion d'interroger l'auteur sur ses motivations personnelles ${ }^{19}$. Jacques DÉOM confie qu'il est fasciné par "la complexité du problème identitaire juif " $^{20}$ et son intérêt porte sur les multiples facettes de cette identité. L'auteur précise ainsi que "ce fragment de l'épopée sioniste, outre son caractère humain, est un aspect du processus fascinant par lequel un groupe tente de se ressaisir pour revenir dans l'histoire $\Perp^{21}$. A cet égard, l'auteur précise que le sionisme marque cette "volonté de réinscrire les Juifs dans l'histoire pour ne plus la subir "22. L'Alyah Bet constitue bel et bien un axe fondamental du mouvement sioniste visant à rassembler les exilés en Palestine puis sur le territoire de l'État d'Israël.

Cet ouvrage a le mérite d'apporter un éclairage important sur I'histoire des Juifs de Belgique. Le récit offert par l'auteur, avec sa dimension romancée et pédagogique, témoigne de l'importance de mieux connaître cette période de l'histoire que ce soit en Belgique

17 DÉOM Jacques, La filière des ombres. L'odyssée des réfugiés juifs Belgique - Palestine (1948-1948), op. cit., p. 51.

${ }^{18}$ DÉOM Jacques, La filière des ombres. L'odyssée des réfugiés juifs Belgique - Palestine (1948-1948), op. cit., p. 167. Maurice SZMIDT a retracé le récit de son aventure dans un ouvrage qui a fait l'objet d'une traduction. Voy. SZMIDT Maurice, Mémoires de déportation, écrits en mai-juin 1945, Paris, Le Manuscrit - Fondation pour la mémoire de la Shoah, 2009, $244 \mathrm{p}$.

19 Jacques DÉOM est déjà l'auteur d'un ouvrage définissant l'antisémitisme. DÉOM Jacques, Qu'est-ce que l'antisémitisme? Quelques éléments de réponse, Bruxelles, Fondation de la Mémoire contemporaine, Bruxelles, 2012, $131 \mathrm{p}$.

${ }^{20}$ Entretien avec Jacques DÉOM, jeudi 28 mai 2015, Bruxelles, Fondation de la Mémoire contemporaine. ${ }^{21} \mathrm{Ibid}$.

${ }^{22}$ Ibid. 
Cahiers

Mémoire et Politique

ou ailleurs ${ }^{23}$, car comme le rappelle pertinemment Jacques DÉOM, « il manque une véritable histoire des Juifs de Belgique au lendemain de la Deuxième Guerre mondiale », bien que quelques travaux existent déjà ${ }^{24}$. Une histoire à poursuivre...

${ }^{23}$ À titre d'information, on se souviendra de la recherche menée par Olivier GuEZ sur le retour des Juifs en Allemagne depuis la fin de la Deuxième Guerre mondiale. Le récit de cet auteur a montré toute la complexité de l'immédiat après-guerre, entre autres, pour de nombreux Juifs ayant choisi de retourner en Allemagne. Guez Olivier, L'impossible retour. Une histoire des Juifs en Allemagne depuis 1945, Paris, Flammarion, 2007, $336 \mathrm{p}$.

${ }^{24}$ Voy. SCHREIBER Jean-Philippe, Dictionnaire biographique des Juifs de Belgique : figures du judaïsme belge, $X I X^{e}-X X^{e}$, Bruxelles, De Boeck et Larcier, 2002, 400 p.; MASSANGE Catherine, Bâtir le lendemain. L'aide aux Israélites victimes de la Guerre et le Service social juif de 1944 à nos jours, Bruxelles, Dedier Devillez, 2002, 219 p. et VANDEN DAELE Veerle, Laten we hun lied verder zingen. De heropbouw van de joodse gemeenschap in Antwerpen na de Tweede Wereldoorlog (1944-1960), Amsterdam, Aksant, 2008, 512 p. 